\title{
Call for awareness of the updated diagnostic criteria and clinical management for patients with tuberous sclerosis complex
}

\section{Alerta para a atualização de Critérios diagnósticos e condutas clínicas para pacientes COM ESCLEROSE TUBEROSA}

Luciana A. Haddad ${ }^{1,2 *}$, Sérgio Rosemberg ${ }^{3}$

${ }^{1}$ Department of Genetics and Evolutionary Biology, Institute of Biosciences, University of São Paulo (USP), São Paulo, SP, Brazil.

${ }^{2}$ Tuberous Sclerosis Alliance International Scientific Advisory Board, Silver Spring, MD, USA.

${ }^{3}$ Department of Pediatrics, Division of Pediatric Neurology, School of Medical Sciences of Santa Casa de São Paulo (FCMSCSP), São Paulo, SP, Brazil.

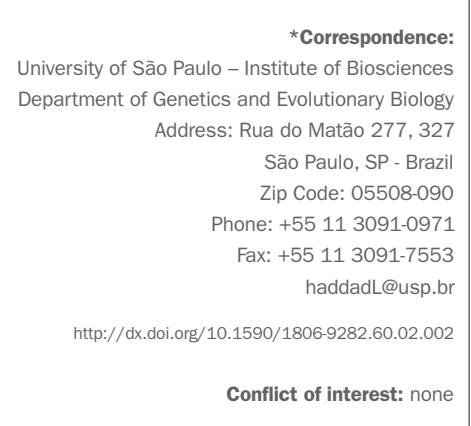

Tuberous sclerosis complex is a multi-system disorder with autosomal dominant inheritance, caused by mutations in either tumor suppressor gene TSC1 or TSC2 (Tuberous Sclerosis Complex 1 or 2). Its clinical diagnosis has been based on the 1998 review of Gómez clinical criteria. The 2012 International Tuberous Sclerosis Complex Consensus Group, comprised of 79 specialists, re-evaluated and updated the clinical criteria, based on a deeper understanding of the disease that took place in the past 15 years. We would like to draw the attention of the reader to the two articles that have been recently published with the recommendations updating the clinical criteria and for the diagnosis, management and longitudinal follow-up of TSC patients $(1,2)$.

TSC diagnosis is basically clinical following the classification of the findings into either major or minor features. Thereafter, three possibilities were possible: definite, probable or possible diagnosis of TSC. The 2012 review regrouped the clinical diagnosis into only two categories, either definite or possible TSC (Table 1). The main alterations are cited as follows. Cortical tubers, previously a major feature, and the minor feature cerebral white matter radial migration lines have been reclassified into a single major feature, cortical dysplasia. Both are due to similar pathologic processes, and may be associated to refractory epilepsy and learning disabilities. Angiomyolipomas are fairly common among TSC patients (approximately 80\%). Although they have frequently a renal location, other organs such as the liver may also be affected. Therefore, at least two angiomyolipomas constitute a major feature, however the renal specification is no longer needed for its classification. Due to the high specificity of cardiac rhabdomyoma to TSC, it remains as a major feature though it is not necessary to specify if single or multiple. Its prenatal or neonatal identification is very important for the diagnosis of the disorder, for which early intervention is more likely to improve the prognosis. More than two intraoral fibromas have replaced the gingival fibromas as a minor feature, since the former comprises buccal, labial and tongue fibromas besides the gingival site. Ungual and periungual fibromas have been reclassified as ungual fibromas, which have lost the post-traumatic designation, and have gained a multiplicity characterization (>2). Dental enamel pits remain a minor feature, currently as three or more pits, although multiplicity or random distribution is no longer necessary for its classification. Shagreen patch has been maintained as a major feature, nevertheless connective tissue nevus is not associated with it because it may also occur isolated or with other disorders. For angiofibroma classification as a major feature, three or more lesions are necessary, as it has been observed isolated in the general population yet in a low frequency. Frontal fibrotic plaques have been replaced by cephalic fibrotic plaque as it comprehends the face and scalp. The non-renal hamartoma minor feature comprehends those from glands, digestive tract and bones. Hamartomatous rectal polyps and bone cysts are 
no longer independent features due to their low specificity for TSC (Table 1).

TABLE 1 Revised Criteria for Tuberous Sclerosis Complex Diagnosis according to the 2012 International Tuberous Sclerosis Complex Consensus Conference ${ }^{1}$

A. Genetic diagnostic criteria

The identification of either a TSC1 or TSC2 pathogenic mutation in DNA from normal tissue is sufficient to make a definite diagnosis of tuberous sclerosis complex (TSC) ${ }^{\text {a }}$.

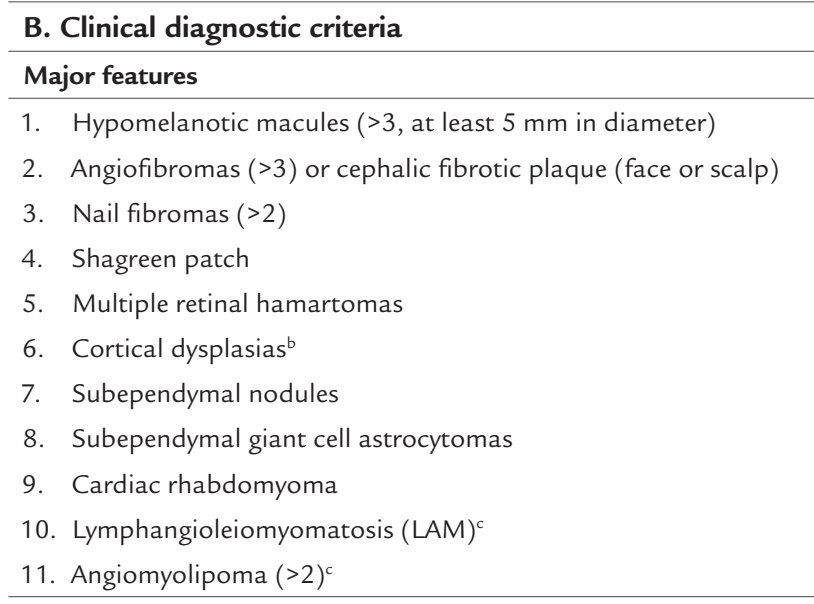

\section{Minor features}

1. Confetti skin lesions

2. Pits in dental enamel $(>3)$

3. Intraoral fibromas $(>2)$

4. Localized retinal achromia

5. Multiple kidney cysts

6. Nonrenal hamartomas

\section{Definite diagnosis:}

Two major features or one major feature with two or more minor features.

\section{Possible diagnosis:}

$\square$ Either one major feature or two or more minor features.

${ }^{a}$ A pathogenic mutation is defined as a mutation that clearly inactivates the function of the TSC1 or TSC2 proteins, prevents protein synthesis or is a missense mutation whose effect on protein function has been established by functional assessment (www.lovd.nl/TSC1, www.lovd/ TSC2)

${ }^{\mathrm{b}}$ Cortical dysplasia includes tubers and cerebral white matter radial migration lines.

c A combination of the two major clinical features (LAM and angiomyolipomas) without other features does not meet criteria for a definite diagnosis.

Besides the review of the clinical diagnosis of TSC, the 2012 panels included its genetic definite diagnosis ( $\mathrm{Ta}$ ble 1). Nearly 75 to $90 \%$ of TSC patients tested by conventional molecular methods have a mutation detected in TSC1 or TSC2 gene. Therefore, although the lack of mutation identified does not exclude TSC, the finding of one pathogenic mutation in TSC1 or TSC2 in normal tissue is at present sufficient for the diagnosis. This may be particularly important for young patients with inconclusive clinical diagnosis who may benefit from early and thorough surveillance of recognized or for novel lesions. By pathogenic mutation it is understood those that impact on protein synthesis or function, such as large genomic deletions; the ones that cause shift in the reading frame for aminoacids; the change of a codon into a stop codon, causing truncated protein; or a missense mutation switching aminoacids. The latter needs experimental functional validation to be considered pathogenic. Any other TSC1 or TSC2 variant whose effect on the protein is uncertain does not meet that criterium. ${ }^{1}$

It is not our goal to summarize here the specific guidelines for surveillance and treatment for lesions in each different organ. Those have been widely explained by Krueger et al (2013). Based on the data available, the 2012 Consensus group has determined four categories of recommendation which have been applied to each aspect evaluated. The recommendation categories were based on the literature study level of evidence and the appropriateness of the diagnostic or therapeutic intervention:

- Category 1: Based upon high-level evidence, there is uniform consensus that the intervention is appropriate;

- Category 2A: Based upon lower-level evidence, there is uniform consensus that the intervention is appropriate;

- Category 2B: Based upon lower-level evidence, there is consensus that the intervention is appropriate;

- Category 3: Based upon any level of evidence, a consensus on appropriate intervention cannot be reached.

Methods and periodicity for surveillance have been described. $^{2}$

The findings that the proteins expressed by the TSC1 and TSC2 genes as a heterodimer have inhibitory roles on the mammalian target of rapamycin (mTOR) led to clinical trials of rapamycin or analogs (mTOR inhibitors) to treat subependymal gyant cell astrocytoma (SEGA), angiomyolipoma and lung lymphangioleiomyomatosis (LAM). In different countries, regulatory agencies have approved the indication of $\mathrm{mTOR}$ inhibitors for distinct TSC lesions. Clinical indications for mTOR inhibition therapy have been reviewed by the consensus group. Clinical conditions that may benefit from short-term mTOR inhibition drugs are: growing, asymptomatic SEGA; asymptomatic, growing, angiomyolipomas larger than 3 
$\mathrm{cm}$ as the first-line therapy; and LAM patients with moderate to severe lung disease or rapid progression. ${ }^{2,3}$ There are certainly other situations where specific clinical aspects of SEGA, angiomyolipoma or LAM patients may confer high surgical risks. Therefore, the risks of both, novel or more conventional therapeutic alternatives should be discussed with the family before making a decision.

Acknowledgements: LAH is grateful to Steve Roberds and Katie Smith (Tuberous Sclerosis Alliance, Silver Spring, MD) for discussion and advices on TSC information outreach.

\section{References}

1. Northrup H, Krueger DA, on behalf of the International Tuberous Sclerosis Complex Consensus Group. Tuberous Sclerosis Complex Diagnostic Criteria Update: Recommendations of the 2012 International Tuberous Sclerosis Complex Consensus Conference. Pediatr Neurol 2013; 49: 243-254.

2. Krueger DA, Northrup $\mathrm{H}$, on behalf of the International Tuberous Sclerosis Complex Consensus Group. Tuberous Sclerosis Complex Surveillance and Management: Recommendations of the 2012 International Tuberous Sclerosis Complex Consensus Conference. Pediatr Neurol 2013; 49:255-265.

3. Roth J, Roach ES, Bartels U, Jóźwiak S, Koenig MK, Weiner HL, Franz DN Wang HZ. Subependymal giant cell astrocytoma: diagnosis, screening, and treatment. Recommendations from the international tuberous sclerosis complex consensus conference 2012. Pediatr Neurol. 2013; 49:439-44. 